\title{
АНАЛИЗ ВЛИЯНИЯ ЛИНЕЙНОГО ТЕМПЕРАТУРНОГО ФАКТОРА И ПОСТОЯННОГО ВДУВА НА ПАРАМЕТРЫ МАТЕМАТИЧЕСКОЙ МОДЕЛИ И ЛОКАЛЬНЫЕ ХАРАКТЕРИСТИКИ ТЕПЛОМАССООБМЕНА И ТРЕНИЯ НА ПРОНИЦАЕМЫХ ПОВЕРХНОСТЯХ ГЛА
}

\author{
Г. Г. Бильченко, Н. Г. Бильченко \\ Казанский Национальный Исследовательский Технический Университет \\ (КНИТУ - КАИ) им. А. Н. Туполева
}

Поступила в редакцию 18.03.2019 г.

\begin{abstract}
Аннотация. Исследуются свойства математической модели управления тепломассообменом и трением в ламинарном пограничном слое на проницаемых цилиндрических и сферических поверхностях гиперзвуковых летательных аппаратов. Рассмотрен случай применения на всём участке управления сочетания линейного температурного фактора и постоянных вдува и магнитного поля. Получены зависимости параметров математической модели, локальных характеристик тепломассообмена и трения, локальной мощности системы, обеспечивающей вдув, от управляющих воздействий. Приведены результаты вычислительных экспериментов.

Ключевые слова: управление, тепломассообмен, ламинарный пограничный слой, гиперзвуковые течения, проницаемые поверхности, линейный температурный фактор, постоянный вдув, локальные характеристики тепломассообмена и трения.
\end{abstract}

\section{ВВЕДЕНИЕ}

Данная работа является расширенным вариантом материала второго [1] из двух докладов, представленных на Международную научно-техническую конференцию АППМИМ-2018 (развёрнутая версия первого доклада [2] изложена в [3]). Работа, сохраняющая все обозначения и сокращения [3], продолжает исследования свойств математической модели ЛПС электропроводящего газа на проницаемых цилиндрических и сферических поверхностях ГЛА и рассматривает влияние следующего сочетания управляющих воздействий: линейного температурного фактора и постоянных вдува и магнитного поля:

1) на параметры математической модели ЛПС $\theta_{0}(x), \theta_{1}(x), \omega_{0}(x), \omega_{1}(x)$;

2) на локальные характеристики ТМО и трения $q(x), f(x)$ и локальную мощность $\eta(x)$ системы, обеспечивающей вдув.

() Бильченко Г. Г., Бильченко Н. Г., 2019

\section{1. ПОСТАНОВКА ЗАДАЧИ}

Аналогично [3] рассмотрим прямую задачу [4]:

$$
\left(m, \tau_{w}, s\right) \rightarrow(q, f, \eta ; Q, F, N) .
$$

Пусть фиксированы значения неизменяемых параметров:

$$
\begin{gathered}
\text { число Маха } M_{\infty} \in[10 ; 40], \\
\text { высота полёта } H \in[10 ; 30][\mathcal{M}], \\
\text { радиус тела } R \in[0,1 ; 1][\mathcal{M}] .
\end{gathered}
$$

Пусть диапазоны изменения управляющих параметров ограничены:

$$
\begin{gathered}
m \in M^{c}=[0 ; 1], \\
\tau_{w} \in T^{c}=[0,15 ; 0,9], \\
\left.s \in S^{c}=\left[0 ; 5 \cdot 10^{4}\right][\text { Tл/(OM } \cdot \mathcal{M})\right] .
\end{gathered}
$$

Далее индекс «w» параметра $\tau_{w}$ и размерность $[$ Тл/(OM $\cdot \mathcal{M})]$ параметра $s$ опущены.

Линейный закон температурного фактора

$$
\begin{gathered}
\tau(x)=\tau\left(x ; \tau_{0}, \tau_{1}\right)= \\
=\tau_{0} \cdot(1-x)+\tau_{1} \cdot x \text { для } x \in X,
\end{gathered}
$$

где $\tau_{0}, \tau_{1} \in T^{c}$, 


\section{Г. Г. Бильченко, Н. Г. Бильченко}

$$
\tau^{\prime}(x)=\tau_{1}-\tau_{0} \in[-0,75 ; 0,75],
$$

при $\tau^{\prime}>0$ является (строго) возрастающим, а при $\tau^{\prime}<0$ - (строго) убывающим. Набор законов (8), соответствующих одинаковому значению $\tau_{0}$ (или $\tau_{1}$ ), назовём пучком по $\tau_{0}$ (по $\tau_{1}$, соответственно).

Для $\tau_{0}, \overline{\tau_{0}}, \tau_{1}, \overline{\tau_{1}} \in T^{c}$ из

$$
\underline{\tau_{0}} \leq \overline{\tau_{0}}
$$

и

$$
\underline{\tau_{1}} \leq \overline{\tau_{1}}
$$

следует

$$
\tau\left(x ; \underline{\tau_{0}}, \underline{\tau_{1}}\right) \leq \tau\left(x ; \overline{\tau_{0}}, \overline{\tau_{1}}\right)
$$

для всех $x \in X$.

Здесь (9), (10) - условия подчинения параметров закона управления (8), а (11) - условие подчинения законов управления.

В условиях применения линейного закона (8) в объединённой системе ОДУ (5)-(8) [4] уравнение (7) примет вид

$$
\begin{gathered}
\omega_{0}^{\prime}=\left(1-\tau_{0}+\left(\tau_{0}-\tau_{1}\right) \cdot x\right) \cdot \theta_{0}^{\prime}+ \\
+\left(\tau_{0}-\tau_{1}\right) \cdot \theta_{0} .
\end{gathered}
$$

Обозначим

$$
\begin{gathered}
T_{05}^{d}=\{0,15 ; 0,2 ; \ldots ; 0,9\} \subset T^{c}, \\
T_{15}^{d}=\{0,15 ; 0,3 ; \ldots ; 0,9\} \subset T_{05}^{d}, \\
M_{50}^{d}=\{0 ; 0,5 ; 1\} \subset M_{25}^{d},
\end{gathered}
$$

где $M_{25}^{d}$ определено в (13) [3]. Элементам множества $T_{05}^{d}$ поставим в соответствие буквы латинского алфавита от « $d »$ до «s». Тогда значение параметра $\tau_{0}$ (или $\tau_{1}$ ) закона температурного фактора (8) из $T_{05}^{d}$ определяется одной буквой, а сам закон - двухбуквенным сочетанием $\{d d, d e, \ldots, s r, s s\}$.

Вычислительные эксперименты выполнены (как в [3]) для воздуха в атмосфере Земли при $H=10[\mathcal{K M}], M_{\infty}=10, R=0,1 \quad[\mathcal{M}]$. Результаты вычислительных экспериментов представлены на рис. 1-15 для сочетания линейного закона температурного фактора (8), где $\tau_{0}, \tau_{1} \in T_{15}^{d}$, a $m(x)=$ Const $\in M_{50}^{d}$ для $\theta_{0}, \theta_{1}$, $\omega_{0}, \omega_{1}, q, f$ и $m(x)=$ Const $\in\{0,25 ; 0,5 ; 0,75\}$ для $\eta$. Матрица обозначений законов температурного фактора приведена в табл. 1. Тогда

1) элементы, расположенные на главной диагонали матрицы, соответствуют постоянным законам температурного фактора;
2) элементы, расположенные выше главной диагонали - законам линейно возрастающего температурного фактора;

3) элементы, расположенные ниже главной диагонали - законам линейно убывающего температурного фактора.

\begin{tabular}{|c||c|c|c|c|c|c|}
\hline$\tau_{1}$ & 0,15 & 0,3 & 0,45 & 0,6 & 0,75 & 0,9 \\
\hline \hline 0,15 & $d d$ & $d g$ & $d j$ & $d m$ & $d p$ & $d s$ \\
\hline 0,3 & $g d$ & $g g$ & $g j$ & $g m$ & $g p$ & $g s$ \\
\hline 0,45 & $j d$ & $j g$ & $j j$ & $j m$ & $j p$ & $j s$ \\
\hline 0,6 & $m d$ & $m g$ & $m j$ & $m m$ & $m p$ & $m s$ \\
\hline 0,75 & $p d$ & $p g$ & $p j$ & $p m$ & $p p$ & $p s$ \\
\hline 0,9 & $s d$ & $s g$ & $s j$ & $s m$ & $s p$ & $s s$ \\
\hline
\end{tabular}

Набор линейных законов температурного фактора, соответствующих сочетаниям с одинаковой первой (или второй) буквой, т.е. указанным в строке (столбце) или в части строки (в части столбца) матрицы, является пучком по $\tau_{0}$ (по $\tau_{1}$, соответственно).

Пример. Наборы сочетаний $\{g d, g g\}$ и $\{g g, g j, g m, g p, g s\}$, а также их объединение $\{g d, \ldots, g s\}$ определяют пучки с $\tau_{0}=0,3$. Наборы сочетаний $\{d m, g m, j m, m m\} \quad$ и $\{m m, p m, s m\}$, а также их объединение $\{d m, \ldots, s m\}$ определяют пучки с $\tau_{1}=0,6$.

Набор графиков (образов) $q$ (или $\left.f, \theta_{0}, \theta_{1}, \omega_{0}, \omega_{1}, \eta\right)$, соответствующих пучку законов температурного фактора (прообразов) при фиксированных $m$ и $s$, также будем называть пучком. Отметим, что кривые, соответствующие пучку по $\tau_{0}$, имеют общую точку при $x=0$ (точку исхода), а у кривых, соответствующих пучку по $\tau_{1}$, точки при $x=1$ обычно различаются.

Для удобства анализа пучки кривых $\theta_{0}, \ldots, \omega_{1}, q, f, \eta$ разделены на две группы: $\tau^{\prime} \geq 0$ и $\tau^{\prime} \leq 0$. Распределение графиков по рисункам приведено в табл. 2.

Таблица 2

\begin{tabular}{|c||c|c|}
\hline & $\tau_{0} \leq \tau_{1}$ & $\tau_{0} \geq \tau_{1}$ \\
\hline \hline$\theta_{0}, \ldots, \omega_{1}$ & рис. 1-3 & рис. $4-6$ \\
\hline \hline$q, f$ & рис. $7-9$ & рис. $10-12$ \\
\hline \hline & рис. 13,14 & рис. 13,15 \\
\hline
\end{tabular}


Анализ влияния линейного температурного фактора и постоянного вдува на параметры ...

С помощью обозначений табл. 1 отметим на рисунках некоторые кривые.

\section{2. ВЛИЯНИЕ НА ПАРАМЕТРЫ МАТЕМАТИЧЕСКОЙ МОДЕЛИ}

Утверждение 1. В условиях (2)-(7) для $k \in\{0 ; 1\}$ и любых постоянных $m \equiv C_{1} \in M^{c}$ u $s \equiv C_{3} \in S^{c}$ в случае применения тинейного закона вдува (8) для любых $\tau_{0}, \overline{\tau_{0}}, \tau_{1}, \overline{\tau_{1}} \in T^{c}$ если выполнены условия (9) и (10), то

$$
\begin{aligned}
& \theta_{k}\left(x ; C_{1}, \tau\left(x ; \underline{\tau_{0}}, \underline{\tau_{1}}\right), C_{3}\right) \geq \\
& \geq \theta_{k}\left(x ; C_{1}, \tau\left(x ; \overline{\tau_{0}}, \overline{\tau_{1}}\right), C_{3}\right), \\
& \omega_{k}\left(x ; C_{1}, \tau\left(x ; \overline{\tau_{0}}, \overline{\tau_{1}}\right), C_{3}\right) \geq \\
& \geq \omega_{k}\left(x ; C_{1}, \tau\left(x ; \overline{\tau_{0}}, \overline{\tau_{1}}\right), C_{3}\right)
\end{aligned}
$$

для всех $x \in X$.

Условия реализации верхних и нижних кривых $\theta_{0}, \ldots, \omega_{1}$ в пучках приведены в табл. 3.

Таблица 3

\begin{tabular}{|c|c||c|c|}
\hline \multicolumn{2}{|c|}{$\theta_{0}(x), \ldots, \omega_{1}(x)$} & \multicolumn{2}{|c|}{ кривая в пучке } \\
\hline случай & пучок & верхняя & нижняя \\
\hline \multirow{2}{*}{$\tau_{0} \leq \tau_{1}$} & по $\tau_{0}$ & $\tau_{1}=\tau_{0}$ & $\max \tau_{1}$ \\
\cline { 2 - 4 } & по $\tau_{1}$ & $\min \tau_{0}$ & $\tau_{0}=\tau_{1}$ \\
\hline \multirow{2}{*}{$\tau_{0} \geq \tau_{1}$} & по $\tau_{0}$ & $\min \tau_{1}$ & $\tau_{1}=\tau_{0}$ \\
\cline { 2 - 4 } & по $\tau_{1}$ & $\tau_{0}=\tau_{1}$ & $\max \tau_{0}$ \\
\hline
\end{tabular}

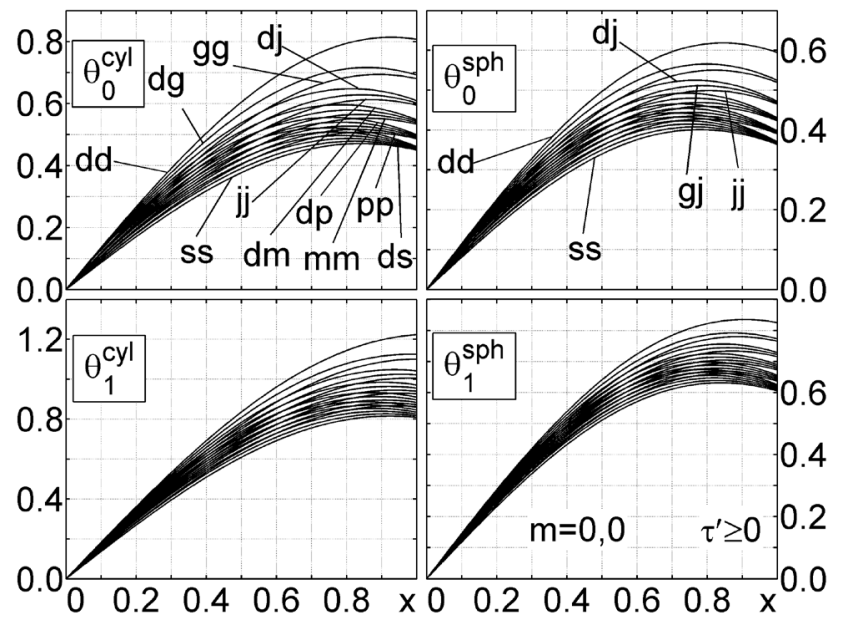

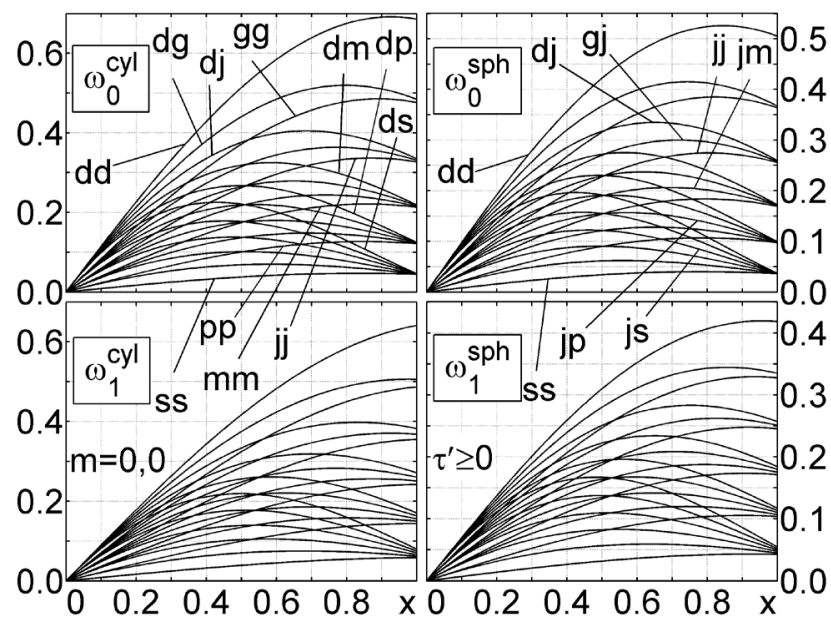

Pис. 1. Зависимости $\theta_{0}(x), \ldots, \omega_{1}(x)$ для линейных $\tau(x)$ при $\tau_{0} \leq \tau_{1}$ u $m=0,0$
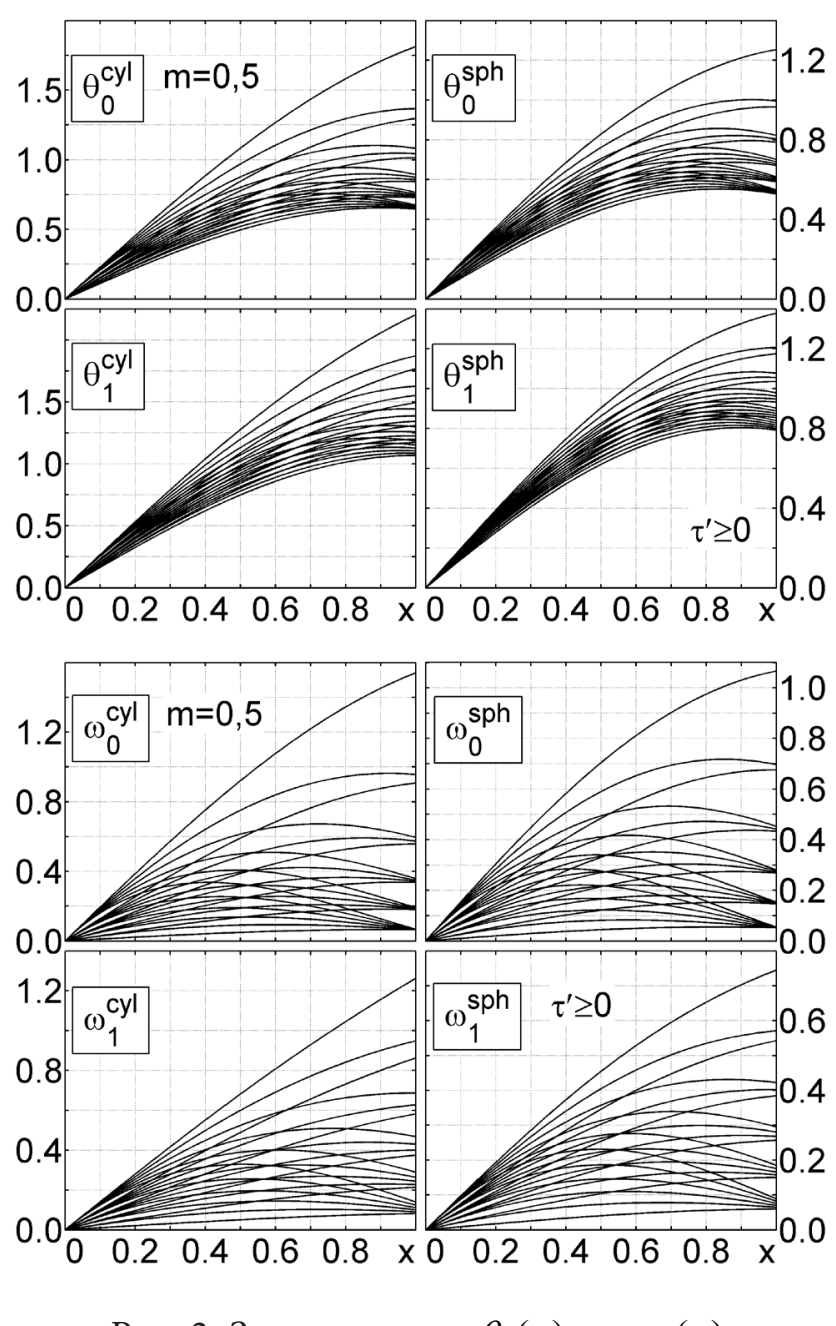

Pис. 2. Зависимости $\theta_{0}(x), \ldots, \omega_{1}(x)$ для линейных $\tau(x)$ при $\tau_{0} \leq \tau_{1} u \quad m=0,5$ 


\section{Г. Г. Бильченко, Н. Г. Бильченко}
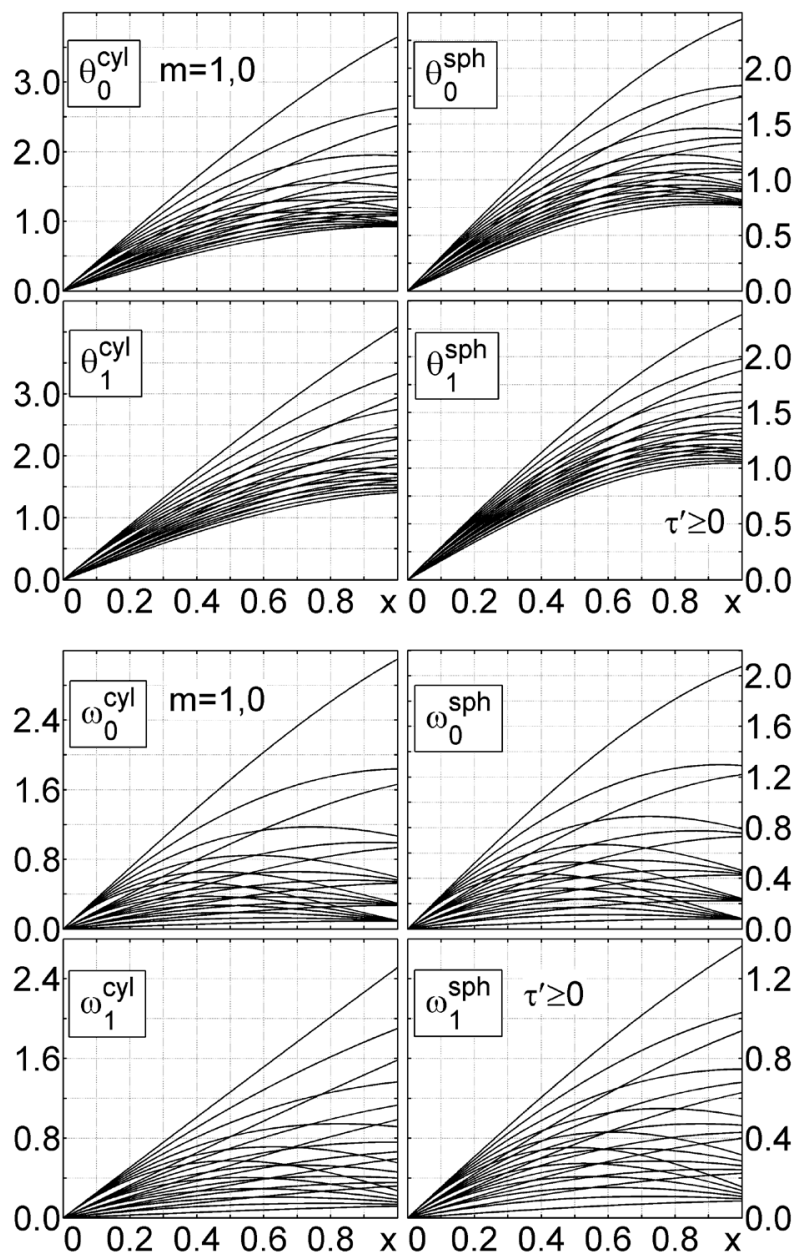

Рис. 3. Зависимости $\theta_{0}(x), \ldots, \omega_{1}(x)$ для линейных $\tau(x)$ при $\tau_{0} \leq \tau_{1} u \quad m=1,0$

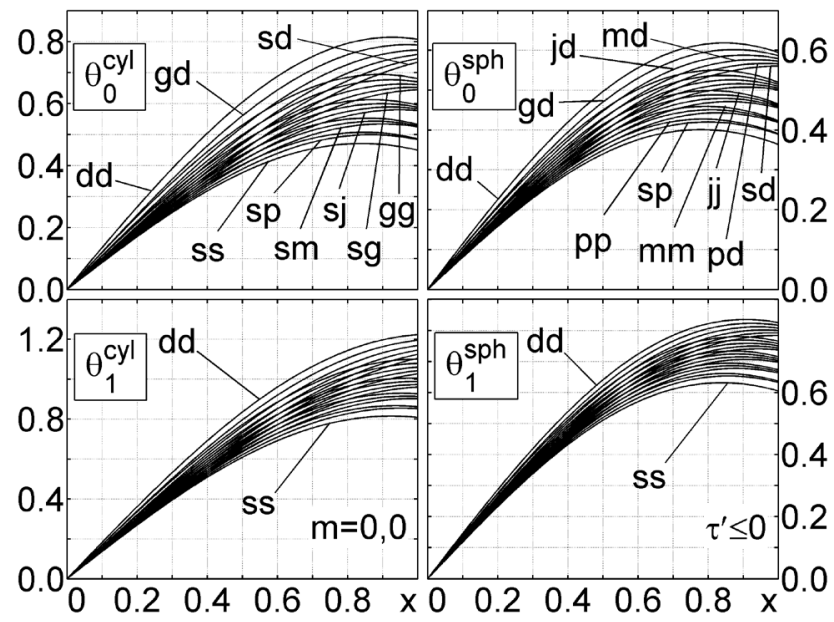

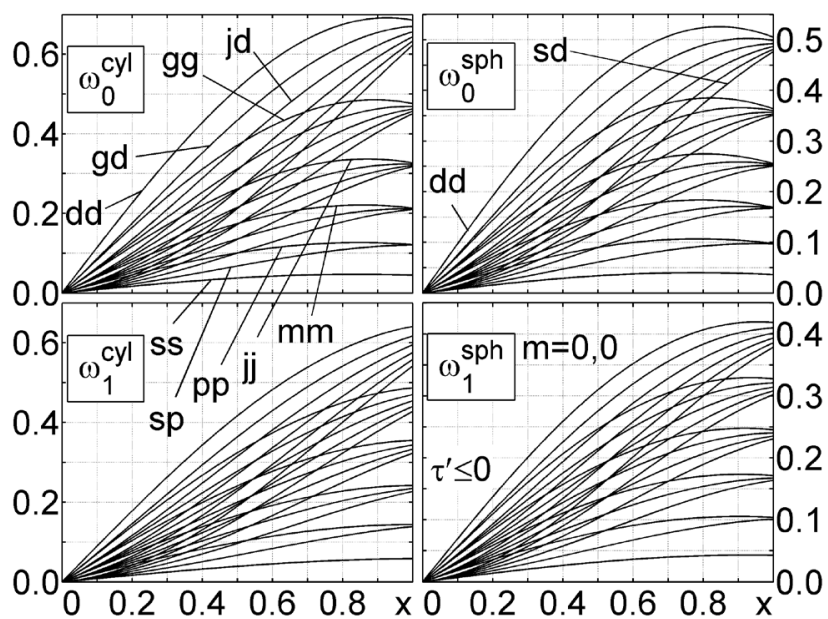

Рис. 4. Зависимости $\theta_{0}(x), \ldots, \omega_{1}(x)$ для линец̆ньх $\tau(x)$ при $\tau_{0} \geq \tau_{1} u m=0,0$
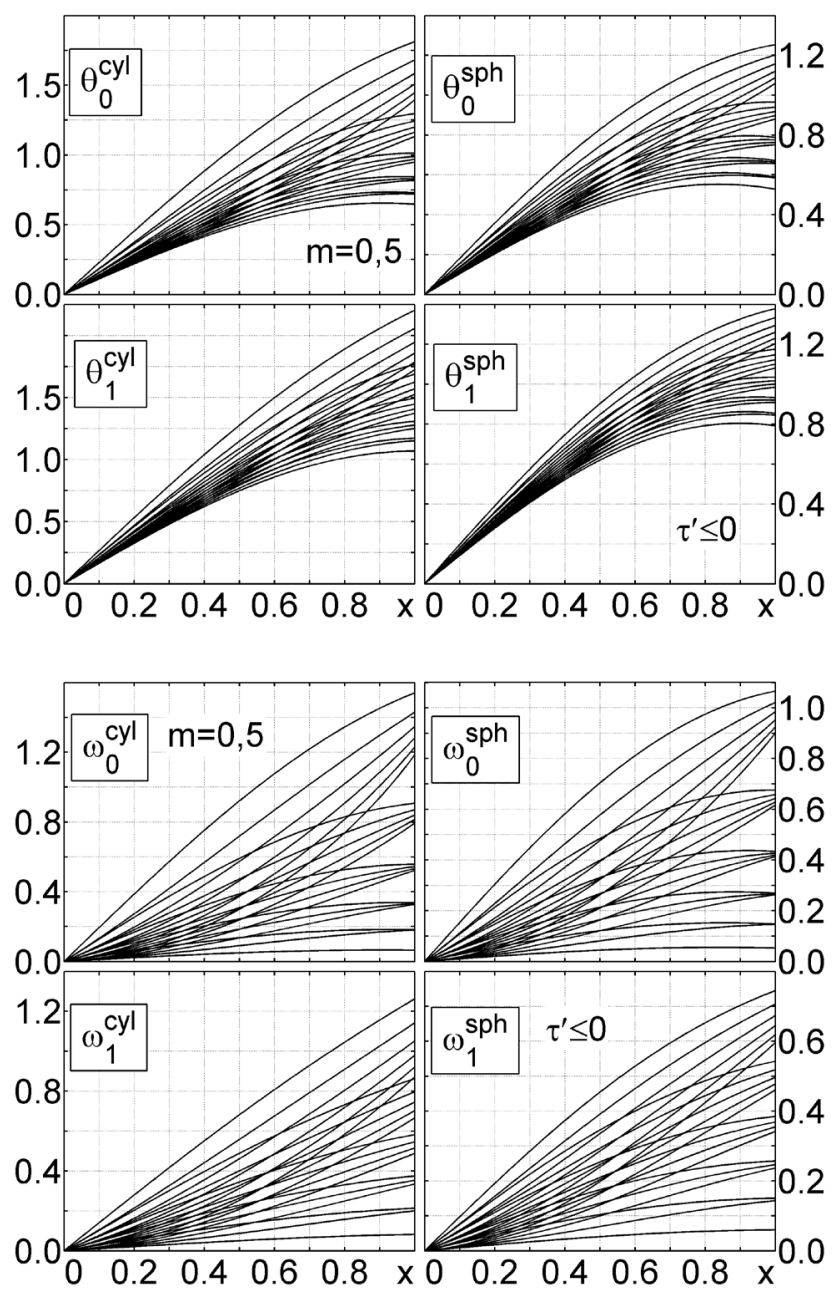

Рис. 5. Зависимости $\theta_{0}(x), \ldots, \omega_{1}(x)$ для тинейньх $\tau(x)$ при $\tau_{0} \geq \tau_{1} u m=0,5$ 
Анализ влияния линейного температурного фактора и постоянного вдува на параметры ...
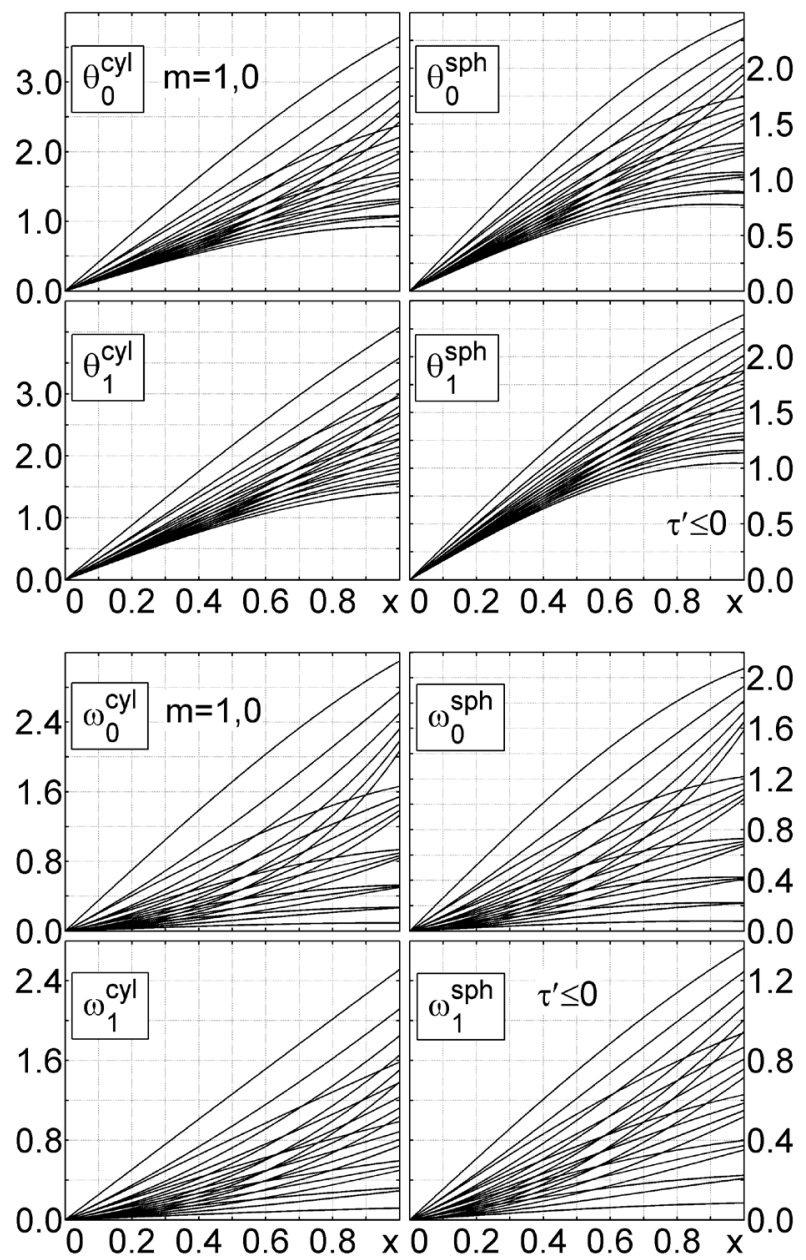

Рис. 6. Зависимости $\theta_{0}(x), \ldots, \omega_{1}(x)$ для линейных $\tau(x)$ при $\tau_{0} \geq \tau_{1}$ и $m=1,0$

\begin{tabular}{|c|c||c|c|}
\hline \multicolumn{2}{|c||}{$q(x)$} & \multicolumn{2}{c|}{ кривая в пучке } \\
\hline случай & пучок & верхняя & нижняя \\
\hline$\tau_{0} \leq \tau_{1}$ & по $\tau_{0}$ & $\tau_{1}=\tau_{0}$ & $\max \tau_{1}$ \\
\hline$\tau_{0} \geq \tau_{1}$ & по $\tau_{0}$ & $\min \tau_{1}$ & $\tau_{1}=\tau_{0}$ \\
\hline
\end{tabular}

Утверждение 3. В условиях (2)-(7) для любых постоянных $m \equiv C_{1} \in M^{c}$ u $s \equiv C_{3} \in S^{c} \quad$ в случае применения тинейного закона (8) для любых $\tau_{0}, \bar{\tau}_{0}, \tau_{1}, \overline{\tau_{1}} \in T^{c}$ если выполнены условия (9) $u(1 \overline{0}), m o$

$$
\begin{aligned}
& f\left(x ; C_{1}, \tau\left(x ; \underline{\tau_{0}}, \underline{\tau_{1}}\right), C_{3}\right) \leq \\
& \leq f\left(x ; C_{1}, \tau\left(x ; \overline{\tau_{0}}, \overline{\tau_{1}}\right), C_{3}\right), \\
& \eta\left(x ; C_{1}, \tau\left(x ; \underline{\tau_{0}}, \underline{\tau_{1}}\right), C_{3}\right) \leq \\
& \leq \eta\left(x ; C_{1}, \tau\left(x ; \overline{\tau_{0}}, \overline{\tau_{1}}\right), C_{3}\right)
\end{aligned}
$$

для всех $x \in X$.

Условия реализации верхних и нижних кривых $f$ и $\eta$ в пучках приведены в табл. 5.

Таблица 5

\begin{tabular}{|c|c||c|c|}
\hline \multicolumn{2}{|c||}{$f(x), \eta(x)$} & \multicolumn{2}{c|}{ кривая в пучке } \\
\hline случай & пучок & верхняя & нижняя \\
\hline \multirow{2}{*}{$\tau_{0} \leq \tau_{1}$} & по $\tau_{0}$ & $\max \tau_{1}$ & $\tau_{1}=\tau_{0}$ \\
\cline { 2 - 4 } & по $\tau_{1}$ & $\tau_{0}=\tau_{1}$ & $\min \tau_{0}$ \\
\hline \multirow{2}{*}{$\tau_{0} \geq \tau_{1}$} & по $\tau_{0}$ & $\tau_{1}=\tau_{0}$ & $\min \tau_{1}$ \\
\cline { 1 - 4 } & по $\tau_{1}$ & $\max \tau_{0}$ & $\tau_{0}=\tau_{1}$ \\
\hline
\end{tabular}

\section{3. ВЛИЯНИЕ НА ПАРАМЕТРЫ ТМО, ТРЕНИЯ И ЛОКАЛЬНУЮ МОЩНОСТЬ СИСТЕМЫ, ОБЕСПЕЧИВАЮЩЕЙ ВДУВ}

Утверждение 2. В условиях (2)-(7) для любых постоянных $m \equiv C_{1} \in M^{c}$ u $s \equiv C_{3} \in S^{c} \quad$ в случае применения тинейного закона (8) для любых $\tau_{0}, \tau_{1}, \overline{\tau_{1}} \in T^{c}$ если выполнено условие (10), mo

$$
\begin{aligned}
& q\left(x ; C_{1}, \tau\left(x ; \tau_{0}, \underline{\tau_{1}}\right), C_{3}\right) \geq \\
& \geq q\left(x ; C_{1}, \tau\left(x ; \tau_{0}, \overline{\tau_{1}}\right), C_{3}\right)
\end{aligned}
$$

для всех $x \in X$.

Условия реализации верхних и нижних кривых $q$ в пучках приведены в табл. 4 .

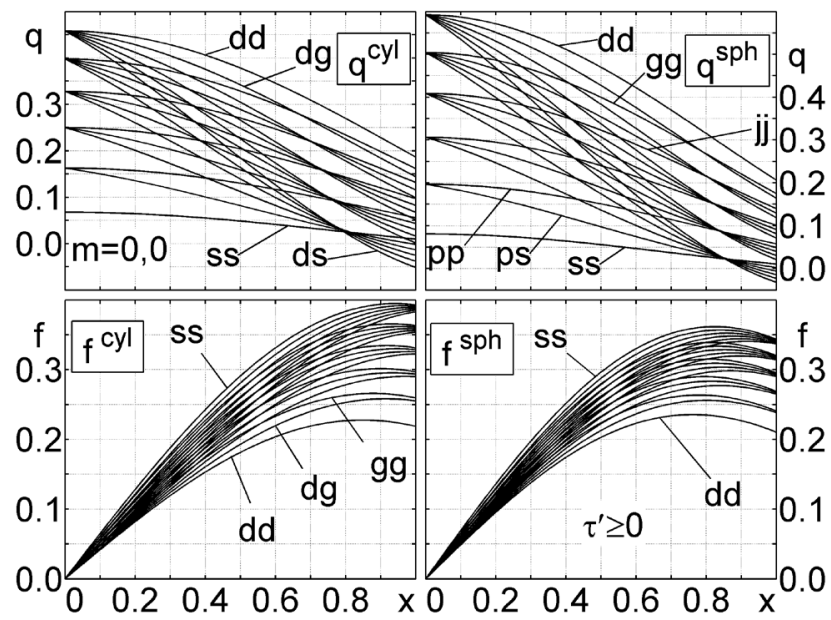

Рис. 7. Зависимости $q(x)$ и $f(x)$ для линейных $\tau(x)$ при $\tau_{0} \leq \tau_{1} u m=0,0$ 


\section{Г. Г. Бильченко, Н. Г. Бильченко}

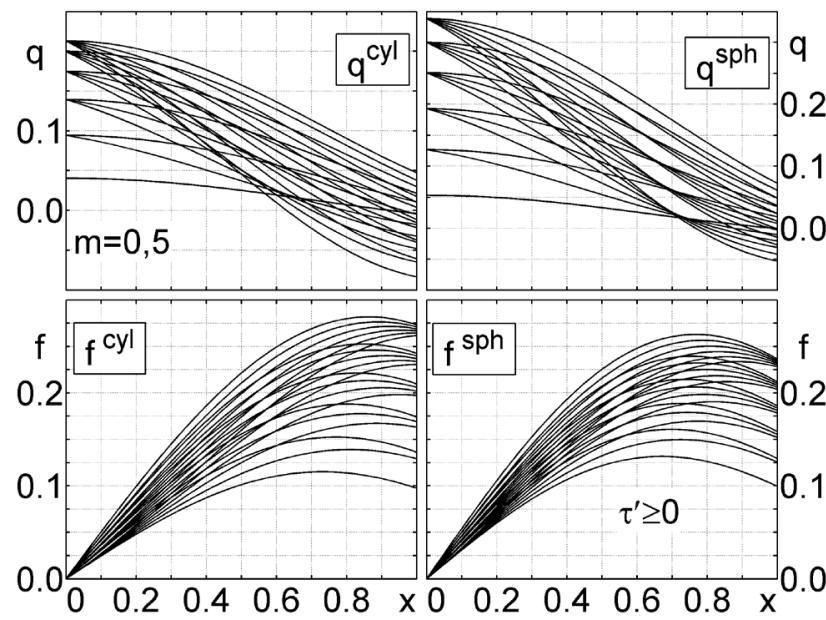

Рис. 8. Зависимости $q(x)$ и $f(x)$

для линейных $\tau(x)$ при $\tau_{0} \leq \tau_{1} u m=0,5$

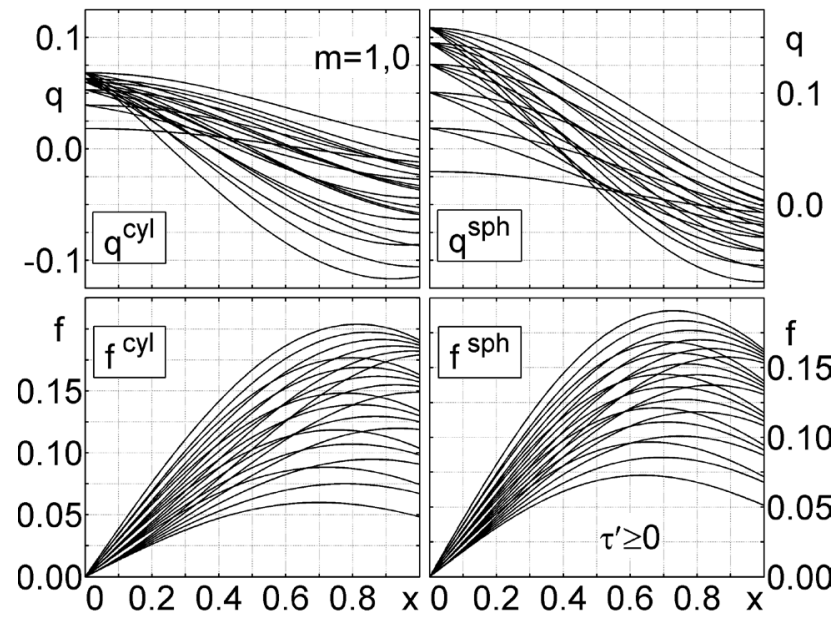

Pис. 9. Зависимости $q(x)$ и $f(x)$

для линейных $\tau(x)$ при $\tau_{0} \leq \tau_{1}$ u $m=1,0$

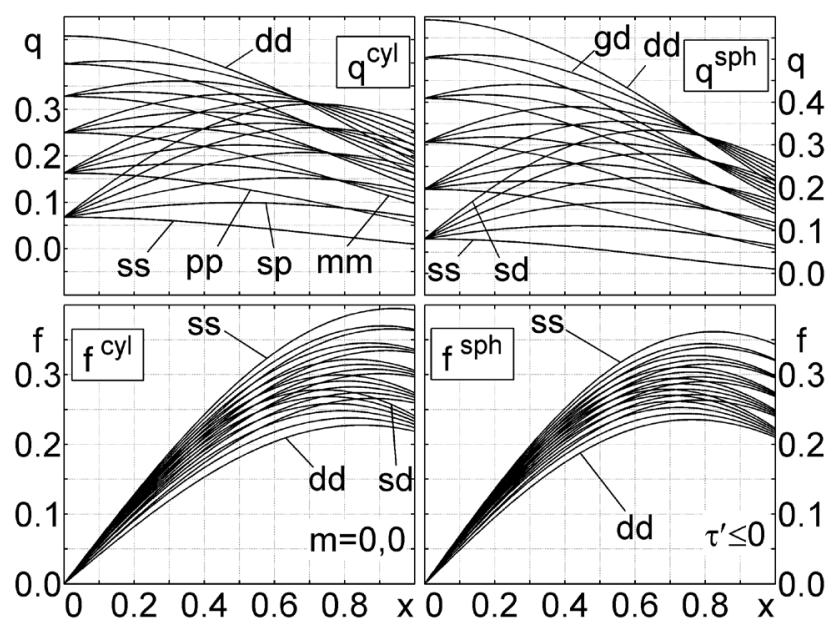

Pис. 10. Зависимости $q(x)$ и $f(x)$ для линейных $\tau(x)$ при $\tau_{0} \geq \tau_{1} u m=0,0$

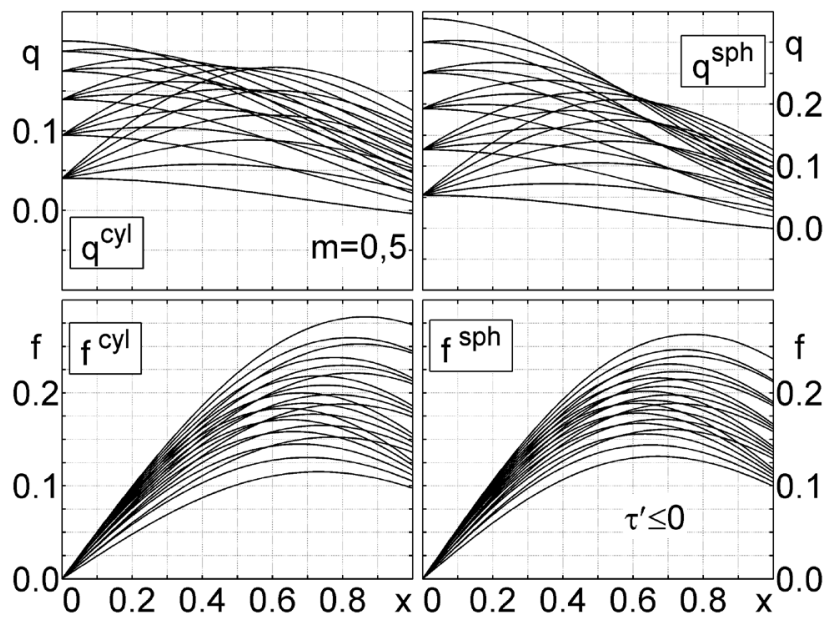

Рис. 11. Зависимости $q(x)$ и $f(x)$ для тинейных $\tau(x)$ при $\tau_{0} \geq \tau_{1} u m=0,5$

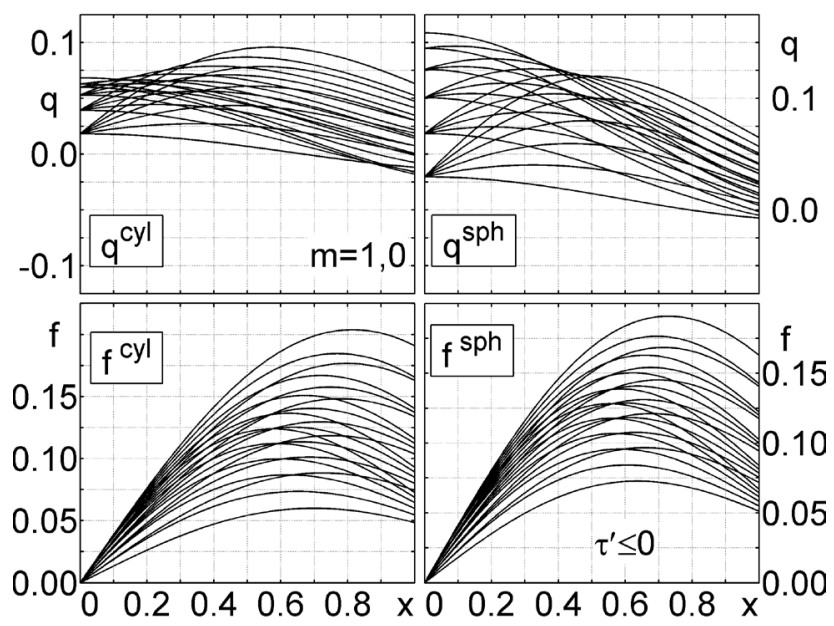

Pис. 12. Зависимости $q(x)$ и $f(x)$

для линейных $\tau(x)$ при $\tau_{0} \geq \tau_{1} u m=1,0$

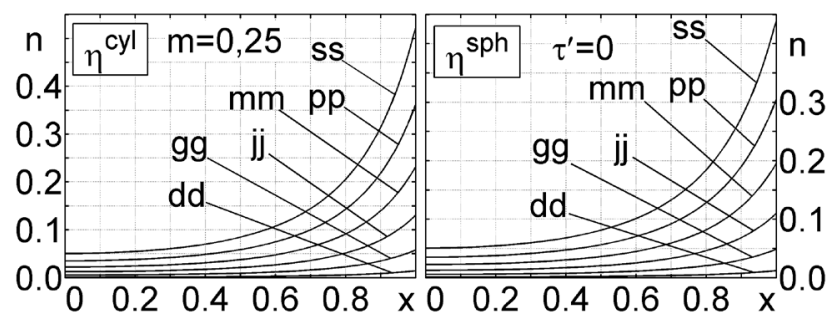

Pис. 13. Мощность $\eta(x)$ для постоянных $\tau(x)$ при $\tau_{0} \in T_{15}^{d}$ u $m=0,25$

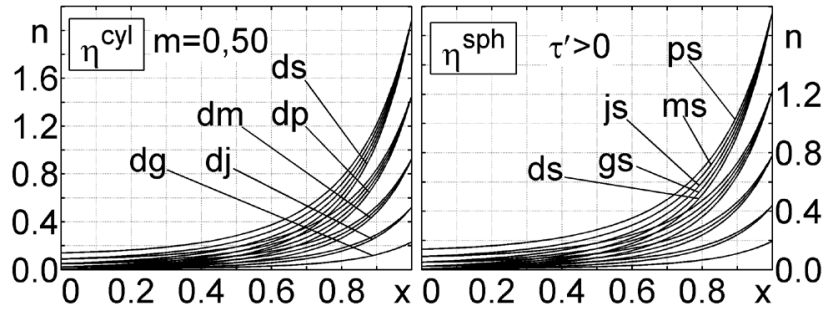

Pис. 14. Мощность $\eta(x)$ для линейных $\tau(x)$ прu $\tau_{0}<\tau_{1} u m=0,5$ 
Анализ влияния линейного температурного фактора и постоянного вдува на параметры ...

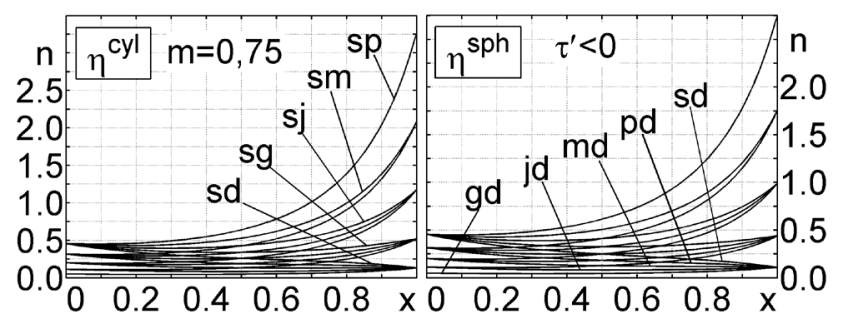

Puс. 15. Мощность $\eta(x)$ для линейных $\tau(x)$ при $\tau_{0}>\tau_{1}$ u $m=0,75$

\section{ЗАКЛЮЧЕНИЕ}

В данной работе изучено влияние на параметры математической модели ламинарного пограничного слоя $\theta_{0}(x), \theta_{1}(x), \omega_{0}(x), \omega_{1}(x)$, на локальные характеристики тепломассообмена и трения $q(x), f(x)$ и локальную мощность $\eta(x)$ системы, обеспечивающей вдув, одного из сочетаний наиболее простых представителей класса монотонных функций линейного температурного фактора и постоянного вдува.

Аналогично [3] полученные результаты вычислительных экспериментов могут быть использованы в качестве моделей наблюдаемых данных в задачах синтеза эффективного управления как на всём участке, так и на его фрагментах.

Анализ влияния сочетания линейного температурного фактора и постоянного вдува на область значений функционалов гиперзвуковой аэродинамики является предметом отдельного исследования.

Бильченко Григорий Григорьевич - канд. физ.-мат. наук, научный сотрудник Лаборатории Моделирования Физико-Технических Процессов (при кафедре Теплотехники и Энергетического Машиностроения) Казанского Национального Исследовательского Технического Университета (КНИТУ-КАИ) им. А. Н. Туполева.

E-mail: ggbil2@gmail.com

\section{СПИСОК ЛИТЕРАТУРЫ}

1.Бильченко, Г.Г. О влиянии линейного температурного фактора при постоянном вдуве на локальные характеристики тепломассообмена и трения на проницаемых поверхностях ГЛА / Г. Г. Бильченко, Н. Г. Бильченко // «Актуальные проблемы прикладной математики, информатики и механики»: Сборник трудов Международной научно-технической конференции, Воронеж, 17-19 декабря 2018 г. - Воронеж : Изд-во «Научно-исследовательские публикации», 2019. - С. 597-604.

2. Бильченко, Г. Г. О влиянии линейного вдува при постоянном температурном факторе на локальные характеристики тепломассообмена и трения на проницаемых поверхностях ГЛА / Г. Г. Бильченко, Н. Г. Бильченко // «Актуальные проблемы прикладной математики, информатики и механики»: Сборник трудов Международной научно-технической конференции, Воронеж, 17-19 декабря 2018 г. - Воронеж : Изд-во «Научно-исследовательские публикации», 2019. - С. 588-596.

3. Бильченко, Г. Г. Анализ влияния линейного вдува и постоянного температурного фактора на параметры математической модели и локальные характеристики тепломассообмена и трения на проницаемых поверхностях ГЛА / Г. Г. Бильченко, Н. Г. Бильченко // Вестник Воронеж. гос. ун-та. Сер. Системный анализ и информационные технологии. 2019. - № 2. - C. 5-14.

4. Бильченко, Г. Г. Анализ влияния постоянных управляющих воздействий на область значений функционалов гиперзвуковой аэродинамики / Г. Г. Бильченко, Н. Г. Бильченко // Вестник Воронеж. гос. ун-та. Сер. Системный анализ и информационные технологии. 2018. - № 2. - C. 5-13.

Бильченко Наталья Григорьевна - канд. физ.-мат. наук, научный сотрудник Лаборатории Моделирования Физико-Технических Процессов (при кафедре Теплотехники и Энергетического Машиностроения) Казанского Национального Исследовательского Технического Университета (КНИТУ-КАИ) им. А. Н. Туполева.

E-mail: bilchnat@gmail.com 


\title{
ANALYSIS OF THE INFLUENCE OF LINEAR TEMPERATURE FACTOR AND CONSTANT BLOWING \\ ON THE PARAMETERS OF THE MATHEMATICAL MODEL AND LOCAL CHARACTERISTICS OF HEAT AND MASS TRANSFER AND FRICTION ON THE PERMEABLE SURFACES OF THE HYPERSONIC AIRCRAFT
}

\begin{abstract}
Bilchenko G. G., Bilchenko N. G.
Kazan National Research Technical University (KNRTU-KAI) named after A. N. Tupolev

Annotation. The properties of mathematical model of heat and mass transfer and friction control in laminar boundary layer on permeable cylindrical and spherical surfaces of hypersonic aircraft are investigated. The case of application of a combination of linear temperature factor and constant blowing and magnetic field for the entire segment of control is considered. The dependences of mathematical model parameters, local heat and mass transfer and friction characteristics, local blowing system power on controls are obtained. The computational experiments results are presented.

Keywords: control, heat and mass transfer, laminar boundary layer, hypersonic flows, permeable surfaces, linear temperature factor, constant blowing, local heat and mass transfer and friction characteristics.
\end{abstract}

Bilchenko Grigorij Grigorievich - Candidate of Science in Physics and Mathematics, Researcher of Laboratory of Modeling of Physical and Technical Processes, Department of Heat Engineering and Power Engineering Machinery, Kazan National Research Technical University (KNRTU-KAI) named after A. N. Tupolev. E-mail: ggbil2@gmail.com
Bilchenko Natalya Grigorievna - Candidate of Science in Physics and Mathematics, Researcher of Laboratory of Modeling of Physical and Technical Processes, Department of Heat Engineering and Power Engineering Machinery, Kazan National Research Technical University (KNRTU-KAI) named after A. N. Tupolev. E-mail: bilchnat@gmail.com 